PROCEEDINGS OF THE

AMERICAN MATHEMATICAL SOCIETY

Volume 140, Number 11, November 2012, Pages 3931-3946

S 0002-9939(2012)11246-8

Article electronically published on March 22, 2012

\title{
TRAVELING WAVES OF THE SPREAD OF AVIAN INFLUENZA
}

\author{
ZHI-CHENG WANG, JIANHONG WU, AND RONGSONG LIU
}

(Communicated by Yingfei Yi)

\begin{abstract}
This paper gives a proof for the existence and nonexistence of traveling wave solutions of a reaction-convection epidemic model for the spatial spread of H5N1 avian influenza involving a wide range of bird species and environmental contamination. The threshold condition for the existence of traveling waves coincides with the basic reproduction number exceeding one. The existence of wave solutions is obtained by constructing an invariant cone of initial functions defined on a large spatial domain, applying a fixed point theorem on this cone and then a limiting argument. The invariant cone is based on the information of initial growth pattern of the epidemic and the final size estimation during the entire course of the outbreak.
\end{abstract}

\section{INTRODUCTION}

We consider the system of reaction diffusion equations

$$
\left\{\begin{array}{l}
\frac{\partial S_{c}}{\partial t}=-\beta_{c} S_{c} V-\alpha_{c} \frac{S_{c} I_{c}}{N_{c}}-v_{c} \frac{\partial S_{c}}{\partial x}+D_{c} \frac{\partial^{2} S_{c}}{\partial x^{2}}, \\
\frac{\partial S_{w}}{\partial t}=-\beta_{w} S_{w} V-\alpha_{e w} \frac{S_{w} E_{w}}{N_{w}}-\alpha_{i w} \frac{S_{w} I_{w}}{N_{w}}-v_{w} \frac{\partial S_{w}}{\partial x}+D_{w} \frac{\partial^{2} S_{w}}{\partial x^{2}}, \\
\frac{\partial I_{c}}{\partial t}=\beta_{c} S_{c} V+\alpha_{c} \frac{S_{c} I_{c}}{N_{c}}-d_{i c} I_{c}-v_{c} \frac{\partial S_{c}}{\partial x}, \\
\frac{\partial E_{w}}{\partial t}=\beta_{w} S_{w} V+\alpha_{e w} \frac{S_{w} E_{w}}{N_{w}}+\alpha_{i w} \frac{S_{w} I_{w}}{N_{w}}-\mu_{w} E_{w}-v_{w} \frac{\partial E_{w}}{\partial x}+D_{w} \frac{\partial^{2} E_{w}}{\partial x^{2}}, \\
\frac{\partial I_{w}}{\partial t}=\mu_{w} E_{w}-d_{i w} I_{w}, \\
\frac{\partial I_{d}}{\partial t}=\beta_{d}\left(N_{d}-I_{d}\right) V+\alpha_{d}\left(N_{d}-I_{d}\right) I_{d} / N_{d}-\gamma_{d} I_{d}-v_{d} \frac{\partial I_{d}}{\partial x}+D_{d} \frac{\partial^{2} I_{d}}{\partial x^{2}}, \\
\frac{\partial V}{\partial t}=r_{c} I_{c}+r_{e w} E_{w}+r_{i w} I_{w}+r_{d} I_{d}-\left(d_{v}+d_{n}\right) V-v_{v} \frac{\partial V}{\partial x}+D_{v} \frac{\partial^{2} V}{\partial x^{2}},
\end{array}\right.
$$

which was introduced in [9] to describe the spatio-temporal spread of H5N1 avian influenza in an ecosystem involving a wide range of bird species: poultry (c), wild birds (w) which are susceptible to and die after H5N1 infection, and wild birds (d) which are susceptible to but can survive after H5N1 infection. Birds are further

Received by the editors January 26, 2010 and, in revised form, May 11, 2011.

2010 Mathematics Subject Classification. Primary 35K57, 92D30; Secondary 34K10.

Key words and phrases. Disease spread, avian influenza, epidemic wave.

The first author was supported in part by the NSF of China (11071105), by FRFCU (lzujbky2011-27) and by the Program for New Century Excellent Talents in University (NCET-2010-0470).

The second author was supported in part by CRC, NSERC, CIHR, MITACS and GEOIDE.

The third author was supported in part by the University of Wyoming's EPScoR start-up fund.

(C)2012 American Mathematical Society Reverts to public domain 28 years from publication 
stratified by their disease status as susceptible $\left(S_{c}, S_{w}\right.$ and $\left.S_{d}\right)$, expected $\left(E_{w}\right)$, and infected $\left(I_{c}, I_{w}\right.$ and $\left.I_{d}\right)$. Note that the $w$ class wild birds can fly some distances even after exposure to the virus, and hence we have included the expected class $E_{w}$. Note also that the total number, $N_{d}$, of $d$ class birds is assumed to be a constant. The model also involves the virus (v) in the environment, and exposed/infected birds may contribute to environmental contamination. In the model, $D_{j}$ and $v_{j}$, with $j=w, d, c, v$, the diffusion and convection coefficients of the category $j$ bird/virus, mass action is used to describe the transmission from the virus in the environment to birds, while the standard incidence is used to describe the transmission between birds $\left(N_{c}=S_{c}+I_{c}, N_{w}=S_{w}+E_{w}+I_{w}\right)$. Finally, the virus production is proportional to the number of infected birds.

The basic reproduction number of the corresponding ODE system is given by $\mathcal{R}_{0}=\rho\left(\mathcal{F} \mathcal{V}^{-1}\right)$, where $\rho$ is the spectral radius of a matrix. The matrices $\mathcal{F}$ and $\mathcal{V}$ are relevant to the linearization of the corresponding ODE system of (1.1) at the disease free state $E_{0}\left(S_{c}, S_{w}, I_{c}, E_{w}, I_{w}, I_{d}, V\right)=\left(S_{c 0}, S_{w 0}, 0,0,0,0,0\right)$ and are given by

$$
\mathcal{F}=\left(\begin{array}{lllll}
\alpha_{c} & 0 & 0 & 0 & \beta_{c} S_{c 0} \\
0 & \alpha_{e w} & \alpha_{i w} & 0 & \beta_{w} S_{w 0} \\
0 & 0 & 0 & 0 & 0 \\
0 & 0 & 0 & \alpha_{d} & \beta_{d} N_{d} \\
r_{c} & r_{e w} & r_{i w} & r_{d} & 0
\end{array}\right), \mathcal{V}=\left(\begin{array}{lllll}
d_{i c} & 0 & 0 & 0 & 0 \\
0 & \mu_{w} & 0 & 0 & 0 \\
0 & -\mu_{w} & d_{i w} & 0 & 0 \\
0 & 0 & 0 & \gamma_{d} & 0 \\
0 & 0 & 0 & 0 & d_{v}+d_{n}
\end{array}\right)
$$

The matrices $\mathcal{F}$ and $\mathcal{V}$ and the reproduction number determine the initial growth pattern of the corresponding ODE system. A preliminary analysis of the model was conducted in [9, where the existence of traveling waves was formally studied and the linkage between the minimal wave speed and the disease propagation rate as well as its implication for the effectiveness of different intervention strategies was described numerically. Here, we provide a rigorous proof for the existence or nonexistence of nontrivial traveling wave solutions depending on the size of the basic reproduction number $\mathcal{R}_{0}$. In addition, we show that when $\mathcal{R}_{0}>1$, there exists an $s^{*}$ such that (1.1) admits a nontrivial traveling wave solution for every wave speed $s>s^{*}$. We refer to [9] for more detailed discussions of the biological relevance of these results.

The basic idea to prove the existence of nontrivial traveling wave solutions is to first construct an appropriately invariant cone of initial functions defined in a large but bounded domain, then apply a fixed point theorem on this cone for the relevant solution operators, and finally to pass to the unbounded spatial domain $\mathbb{R}$ by a limiting argument. This method is motivated by [4, 5], where the existence and nonexistence of traveling wave solutions for some infection-age structured epidemic models with diffusion are studied. Adaptation of the approach developed in [4, 5, to our model is highly nontrivial, as the multiple bird species and virus involved make the construction of an invariant cone very difficult. Here we successfully construct such a cone by using the initial growth pattern and the final size outbreak which are encoded by the matrices $\mathcal{F}$ and $\mathcal{V}$, the reproduction number, and the spatial diffusion. Relevant to our work here are [8, 10, and we hope our work provides a framework for more general diffusive epidemic models with species diversity. 


\section{NONEXISTENCE OF TRAVELING WAVE SOLUTIONS}

A traveling wave of (1.1) is a solution with the form $\left(S_{c}(x+s t), I_{c}(x+s t)\right.$, $\left.S_{w}(x+s t), E_{w}(x+s t), I_{w}(x+s t), I_{d}(x+s t), V(x+s t)\right)$. So, with the wave variable $\xi=x+s t$, we have

$$
\left\{\begin{array}{l}
\left(s+v_{c}\right) S_{c}^{\prime}=-\beta_{c} S_{c} V-\alpha_{c} S_{c} I_{c} / N_{c}+D_{c} S_{c} \\
\left(s+v_{w}\right) S_{w}^{\prime}=-\beta_{w} S_{w} V-\alpha_{e w} S_{w} E_{w} / N_{w}-\alpha_{i w} S_{w} I_{w} / N_{w}+D_{w} S_{w}^{\prime \prime} \\
\left(s+v_{c}\right) I_{c}^{\prime}=\beta_{c} S_{c} V+\alpha_{c} S_{c} I_{c} / N_{c}-d_{i c} I_{c} \\
\left(s+v_{w}\right) E_{w}^{\prime}=\beta_{w} S_{w} V+S_{w}\left[\alpha_{e w} E_{w}+\alpha_{i w} I_{w}\right] / N_{w}-\mu_{w} E_{w}+D_{w} E_{w}^{\prime \prime} \\
s I_{w}^{\prime}=\mu_{w} E_{w}-d_{i w} I_{w} \\
\left(s+v_{d}\right) I_{d}^{\prime}(\xi)=\beta_{d}\left(N_{d}-I_{d}\right) V+\alpha_{d}\left(N_{d}-I_{d}\right) I_{d} / N_{d}-\gamma_{d} I_{d}+D_{d} I_{d}^{\prime \prime} \\
\left(s+v_{v}\right) V^{\prime}=r_{c} I_{c}+r_{e w} E_{w}+r_{i w} I_{w}+r_{d} I_{d}-\left(d_{v}+d_{n}\right) V+D_{v} V^{\prime \prime}
\end{array}\right.
$$

Theorem 2.1. Assume that $\mathcal{R}_{0}=\rho\left(\mathcal{F} \mathcal{V}^{-1}\right)<1$. Then for any $c>0$, the trivial solution $\left(S_{c} \equiv S_{c 0}, S_{w} \equiv S_{w 0}, I_{c} \equiv 0, E_{w} \equiv 0, I_{w} \equiv 0, I_{d} \equiv 0, V \equiv 0\right)$ is the unique nonnegative and bounded solution of (2.1) satisfying

$$
\begin{gathered}
S_{c}(-\infty)=S_{c 0}, S_{w}(-\infty)=S_{w 0}, \\
I_{c}(-\infty)=E_{w}(-\infty)=I_{w}(-\infty)=I_{d}(-\infty)=V(-\infty)=0 .
\end{gathered}
$$

Proof. Note that if $\left(I_{c}, E_{w}, I_{w}, I_{d}, V\right) \equiv \mathbf{0}$, then $S_{c} \equiv S_{c 0}$ and $S_{w} \equiv S_{w 0}$. Assume that (2.2) and (2.3) hold and $\left(I_{c}, E_{w}, I_{w}, I_{d}, V\right)$ is not identically zero. An application of the fluctuation lemma ([7]) yields that $S_{c}^{\prime}(-\infty)=S_{w}^{\prime}(-\infty)=0$. Consequently, we can show that $S_{c}^{\prime}(x) \leq 0$ and $S_{w}^{\prime}(x) \leq 0$ for $x \in \mathbb{R}$. Thus, we have $S_{c}(x) \leq S_{c 0}$ and $S_{w}(x) \leq S_{w 0}$ for $x \in \mathbb{R}$. In particular, we have $I_{d}(x) \leq N_{d}$ for $x \in \mathbb{R}$. Note that the system of equations for $\left(I_{c}, E_{w}, I_{w}, I_{d}, V\right)$ is equivalent to

$$
\left\{\begin{array}{l}
I_{c}(\xi)=\int_{-\infty}^{\xi} \frac{d_{i c}}{s+v_{c}} e^{-\frac{d_{i c}}{s+v_{c}}(\xi-t)} \frac{1}{d_{i c}}\left[\beta_{c} S_{c}(t) V(t)+\alpha_{c} S_{c}(t) I_{c}(t) / N_{c}(t)\right] d t, \\
E_{w}(\xi)=\int_{-\infty}^{\xi} \frac{\mu_{w}}{\rho_{w}} e^{\lambda_{w}^{-}(\xi-t)} \frac{1}{\mu_{w}} H_{w}(t) d t+\int_{\xi}^{\infty} \frac{\mu_{w}}{\rho_{w}} e^{\lambda_{w}^{+}(\xi-t)} \frac{1}{\mu_{w}} H_{w}(t) d t, \\
I_{w}(\xi)=\int_{-\infty}^{\xi} \frac{d_{i w}}{s} e^{-\frac{d_{i w}}{s}(\xi-t) \frac{\mu_{w}}{d_{i w}} E_{w}(t) d t} \\
I_{d}(\xi)=\int_{-\infty}^{\xi} \frac{\gamma_{d}}{\rho_{d}} e^{\lambda_{d}^{-}(\xi-t)} \frac{1}{\gamma_{d}} H_{d}(t) d t+\int_{\xi}^{\infty} \frac{\gamma_{d}}{\rho_{d}} e^{\lambda_{d}^{+}(\xi-t)} \frac{1}{\gamma_{d}} H_{d}(t) d t \\
V(\xi)=\int_{-\infty}^{\xi} \frac{d_{v}+d_{n}}{\rho_{v}} e^{\lambda_{v}^{-}(\xi-t)} \frac{1}{d_{v}+d_{n}} H_{v}(t) d t+\int_{\xi}^{\infty} \frac{d_{v}+d_{n}}{\rho_{v}} e^{\lambda_{v}^{+}(\xi-t)} \frac{1}{d_{v}+d_{n}} H_{v}(t) d t,
\end{array}\right.
$$

where

$$
\begin{aligned}
& \lambda_{w}^{ \pm}=\frac{\left(s+v_{w}\right) \pm \sqrt{\left(s+v_{w}\right)^{2}+4 D_{w} \mu_{w}}}{2 D_{w}}, \quad \lambda_{d}^{ \pm}=\frac{\left(s+v_{d}\right) \pm \sqrt{\left(s+v_{d}\right)^{2}+4 D_{d} \gamma_{d}}}{2 D_{d}}, \\
& \lambda_{v}^{ \pm}=\frac{\left(s+v_{v}\right) \pm \sqrt{\left(s+v_{v}\right)^{2}+4 D_{v}\left(d_{v}+d_{n}\right)}}{2 D_{v}} \\
& \rho_{w}=\lambda_{w}^{+}-\lambda_{w}^{-}, \quad \rho_{d}=\lambda_{d}^{+}-\lambda_{d}^{-}, \quad \rho_{v}=\lambda_{v}^{+}-\lambda_{v}^{-} \\
& H_{w}(t):=\beta_{w} S_{w}(t) V(t)+\alpha_{e w} S_{w}(t) E_{w}(t) / N_{w}(t)+\alpha_{i w} S_{w}(t) I_{w}(t) / N_{w}(t), \\
& H_{d}(t):=\beta_{d}\left(N_{d}-I_{d}(t)\right) V(t)+\alpha_{d}\left(N_{d}-I_{d}(t)\right) I_{d}(t) / N_{d} \\
& H_{v}(t):=r_{c} I_{c}(t)+r_{e w} E_{w}(t)+r_{i w} I_{w}(t)+r_{d} I_{d}(t) .
\end{aligned}
$$


Therefore,

$$
\left\{\begin{array}{l}
I_{c}(\xi) \leq \int_{-\infty}^{\xi} \frac{d_{i c}}{s+v_{c}} e^{-\frac{d_{i c}}{s+v_{c}}(\xi-t)} \frac{1}{d_{i c}}\left[\beta_{c} S_{c 0} V(t)+\alpha_{c} I_{c}(t)\right] d t \\
E_{w}(\xi) \leq \int_{-\infty}^{\xi} \frac{\mu_{w}}{\rho_{w}} e^{\lambda_{w}^{-}(\xi-t)} \frac{1}{\mu_{w}}\left[\beta_{w} S_{w 0} V(t)+\alpha_{e w} E_{w}(t)+\alpha_{i w} I_{w}(t)\right] d t \\
\quad \quad+\int_{\xi}^{\infty} \frac{\mu_{w}}{\rho_{w}} e^{\lambda_{w}^{+}(\xi-t)} \frac{1}{\mu_{w}}\left[\beta_{w} S_{w 0} V(t)+\alpha_{e w} E_{w}(t)+\alpha_{i w} I_{w}(t)\right] d t \\
I_{w}(\xi)=\int_{-\infty}^{\xi} \frac{d_{i w}}{s} e^{-\frac{d_{i w}}{s}(\xi-t) \frac{\mu_{w}}{d_{i w}} E_{w} d t} \\
I_{d}(\xi) \leq \int_{-\infty}^{\xi} \frac{\gamma_{d}}{\rho_{d}} e^{\lambda_{d}^{-}(\xi-t)} \frac{1}{\gamma_{d}}\left[\beta_{d} N_{d} V(t)+\alpha_{d} I_{d}(t)\right] d t \\
\quad+\int_{\xi}^{\infty} \frac{\gamma_{d}}{\rho_{d}} e^{\lambda_{d}^{+}(\xi-t)} \frac{1}{\gamma_{d}}\left[\beta_{d} N_{d} V(t)+\alpha_{d} I_{d}(t)\right] d t \\
V(\xi)=\int_{-\infty}^{\xi} \frac{d_{v}+d_{n}}{\rho_{v}} e^{\lambda_{v}^{-}(\xi-t)} \frac{1}{d_{v}+d_{n}} H_{v}(t) d t+\int_{\xi}^{\infty} \frac{d_{v}+d_{n}}{\rho_{v}} e^{\lambda_{v}^{+}(\xi-t)} \frac{1}{d_{v}+d_{n}} H_{v}(t) d t .
\end{array}\right.
$$

Namely,

$$
\left\{\begin{array}{l}
I_{c}(\xi) \leq\left(\mathcal{V}^{-1} \mathcal{F}\right)_{1} \int_{-\infty}^{\xi} \frac{d_{i c}}{s+v_{c}} e^{-\frac{d_{i c}}{s+v_{c}}(\xi-t)} N(t) d t \\
E_{w}(\xi) \leq\left(\mathcal{V}^{-1} \mathcal{F}\right)_{2}\left[\int_{-\infty}^{\xi} \frac{\mu_{w}}{\rho_{w}} e^{\lambda_{w}^{-}(\xi-t)} N(t) d t+\int_{\xi}^{\infty} \frac{\mu_{w}}{\rho_{w}} e^{\lambda_{w}^{+}(\xi-t)} N(t) d t\right], \\
I_{w}(\xi) \leq\left(\mathcal{V}^{-1} \mathcal{F}\right)_{3}\left[\int_{-\infty}^{\xi} \frac{d_{i w}}{s} e^{-\frac{d_{i w}}{s}(\xi-t)} \int_{-\infty}^{t} \frac{\mu_{w}}{\rho_{w}} e^{\lambda_{w}^{-}(t-s)} N(s) d s d t\right. \\
\left.\quad+\int_{-\infty}^{\xi} \frac{d_{i w}}{s} e^{-\frac{d_{i w}}{s}(\xi-t)} \int_{t}^{\infty} \frac{\mu_{w}}{\rho_{w}} e^{\lambda_{w}^{+}(t-s)} N(s) d s d t\right], \\
I_{d}(\xi) \leq\left(\mathcal{V}^{-1} \mathcal{F}\right)_{4}\left[\int_{-\infty}^{\xi} \frac{\gamma_{d}}{\rho_{d}} e^{\lambda_{d}^{-}(\xi-t)} N(t) d t+\int_{\xi}^{\infty} \frac{\gamma_{d}}{\rho_{d}} e^{\lambda_{d}^{+}(\xi-t)} N(t) d t\right], \\
V(\xi) \leq\left(\mathcal{V}^{-1} \mathcal{F}\right)_{5}\left[\int_{-\infty}^{\xi} \frac{d_{v}+d_{n}}{\rho_{v}} e^{\lambda_{v}^{-}(\xi-t)} N(t) d t+\int_{\xi}^{\infty} \frac{d_{v}+d_{n}}{\rho_{v}} e^{\lambda_{v}^{+}(\xi-t)} N(t) d t\right],
\end{array}\right.
$$

where $\left(\mathcal{V}^{-1} \mathcal{F}\right)_{i}$ denotes the $i$-th row of the matrix $\mathcal{V}^{-1} \mathcal{F}$ and

$$
\mathcal{V}^{-1} \mathcal{F}=\left(\begin{array}{lllll}
\frac{\alpha_{c}}{d_{i c}} & 0 & 0 & 0 & \frac{\beta_{c} S_{c 0}}{d_{i c}} \\
0 & \frac{\alpha_{e w}}{\mu_{w}} & \frac{\alpha_{i w}}{\mu_{w}} & 0 & \frac{\beta_{w} S_{w 0}}{\mu_{w}} \\
0 & \frac{\alpha_{e w}}{d_{i w}} & \frac{\alpha_{i w}}{d_{i w}} & 0 & \frac{\beta_{w} S_{w 0}}{d_{i w}} \\
0 & 0 & 0 & \frac{\alpha_{d}}{\gamma_{d}} & \frac{\beta_{d} N_{d}}{\gamma_{d}} \\
\frac{r_{c}}{d_{v}+d_{n}} & \frac{r_{e w}}{d_{v}+d_{n}} & \frac{r_{i w}}{d_{v}+d_{n}} & \frac{r_{d}}{d_{v}+d_{n}} & 0
\end{array}\right), \quad N(t)=\left(\begin{array}{l}
I_{c}(t) \\
E_{w}(t) \\
I_{w}(t) \\
I_{d}(t) \\
V(t)
\end{array}\right)
$$

Let $\sup _{\xi \in \mathbb{R}} I_{c}(\xi)=I_{c}^{0}, \sup _{\xi \in \mathbb{R}} E_{w}(\xi)=E_{w}^{0}, \sup _{\xi \in \mathbb{R}} I_{w}(\xi)=I_{w}^{0}, \sup _{\xi \in \mathbb{R}} I_{w}(\xi)=$ $I_{d}^{0}$ and $\sup _{\xi \in \mathbb{R}} V(\xi)=V^{0}$. Then $N^{0}:=\left(I_{c}^{0}, E_{w}^{0}, I_{w}^{0}, I_{d}^{0}, V^{0}\right)^{T} \geq \mathbf{0}$ and $N^{0} \neq \mathbf{0}$, where $T$ is the transpose. Furthermore, we have

$$
N^{0} \leq\left(\mathcal{V}^{-1} \mathcal{F}\right) N^{0}
$$

If $\rho\left(\mathcal{F} \mathcal{V}^{-1}\right):=\rho_{0}<1$, then there exists a nontrivial vector $P:=\left(p_{1}, p_{2}, 0, p_{4}, p_{5}\right) \geq$ 0 (page 16, Theorem 3.5 of [3]) such that $\left(\mathcal{F V}^{-1}\right) P=\rho_{0} P$. It is easy to verify that $p_{1}>0, p_{2}>0, p_{4}>0, p_{5}>0$. Then $\mathcal{V}^{-1} P>0$ and there holds $\left(\mathcal{V}^{-1} \mathcal{F}\right)\left(\mathcal{V}^{-1} P\right)=$ $\mathcal{V}^{-1}\left(\mathcal{F V}^{-1}\right) P=\rho_{0} \mathcal{V}^{-1} P$, which implies that $\rho_{0}<1$ is a nonnegative engeinvalue of the matrix $\mathcal{V}^{-1} \mathcal{F}$ with positive engeinvector $\mathcal{V}^{-1} P$. It is easy to show that $\mathcal{V}^{-1} \mathcal{F}$ is irreducible, that is, $\left(\mathcal{V}^{-1} \mathcal{F}+\mathcal{I}\right)^{4}>0$. Then the Perron-Frobenius theorem yields that $\rho\left(\mathcal{V}^{-1} \mathcal{F}\right)=\rho_{0}<1$. So, iterating (2.4) yields $N^{0}=\mathbf{0}$, a contradiction. 


\section{EXISTENCE OF TRAVELING WAVE SOLUTIONS}

In the following, we prove the existence of traveling waves of (1.1) when $\mathcal{R}_{0}>1$. Linearizing (2.1) for $I_{c}, E_{w}, I_{w}, I_{d}$ and $V$ in the region $\xi \rightarrow-\infty$ where $S_{c} \rightarrow S_{c 0}$, $S_{w} \rightarrow S_{w 0}$, and setting the remaining variables approaching zero, we have

$$
\left\{\begin{array}{l}
s I_{c}^{\prime}(\xi)=\beta_{c} S_{c 0} V+\alpha_{c} I_{c}-d_{i c} I_{c}-v_{c} I_{c}^{\prime}(\xi) \\
s E_{w}^{\prime}(\xi)=\beta_{w} S_{w 0} V+\alpha_{e w} E_{w}+\alpha_{i w} I_{w}-\mu_{w} E_{w}-v_{w} E_{w}^{\prime}(\xi)+D_{w} E_{w}^{\prime \prime}(\xi) \\
s I_{w}^{\prime}(\xi)=\mu_{w} E_{w}-d_{i w} I_{w} \\
s I_{d}^{\prime}(\xi)=\beta_{d} N_{d} V+\alpha_{d} I_{d}-\gamma_{d} I_{d}-v_{d} I_{d}^{\prime}(\xi)+D_{d} I_{d}^{\prime \prime}(\xi) \\
s V^{\prime}(\xi)=r_{c} I_{c}+r_{e w} E_{w}+r_{i w} I_{w}+r_{d} I_{d}-\left(d_{v}+d_{n}\right) V-v_{v} V^{\prime}(\xi)+D_{v} V^{\prime \prime}(\xi) .
\end{array}\right.
$$

Looking for the solutions of the form $\left(I_{c}, E_{w}, I_{w}, I_{d}, V\right)=\left(q_{1}, q_{2}, q_{3}, q_{4}, q_{5}\right) e^{\lambda \xi}$, where $q_{i}>0$ and $\lambda>0$, we have

$$
\left\{\begin{array}{l}
s \lambda q_{1}=\beta_{c} S_{c 0} q_{5}+\alpha_{c} q_{1}-d_{i c} q_{1}-v_{c} \lambda q_{1} \\
s \lambda q_{2}=\beta_{w} S_{w 0} q_{5}+\alpha_{e w} q_{2}+\alpha_{i w} q_{3}-\mu_{w} q_{2}-v_{w} \lambda q_{2}+D_{w} \lambda^{2} q_{2} \\
s \lambda q_{3}=\mu_{w} q_{2}-d_{i w} q_{3} \\
s \lambda q_{4}=\beta_{d} N_{d} q_{5}+\alpha_{d} q_{4}-\gamma_{d} q_{4}-v_{d} \lambda q_{4}+D_{d} \lambda^{2} q_{4} \\
s \lambda q_{5}=r_{c} q_{1}+r_{e w} q_{2}+r_{i w} q_{3}+r_{d} q_{4}-\left(d_{v}+d_{n}\right) q_{5}-v_{v} \lambda q_{5}+D_{v} \lambda^{2} q_{5}
\end{array}\right.
$$

Let

$$
\widetilde{A}=\left(\begin{array}{lllll}
0 & 0 & 0 & 0 & 0 \\
0 & D_{w} & 0 & 0 & 0 \\
0 & 0 & 0 & 0 & 0 \\
0 & 0 & 0 & D_{d} & 0 \\
0 & 0 & 0 & 0 & D_{v}
\end{array}\right), \widetilde{B}=\left(\begin{array}{lllll}
s+v_{c} & 0 & 0 & 0 & 0 \\
0 & s+v_{w} & 0 & 0 & 0 \\
0 & 0 & s & 0 & 0 \\
0 & 0 & 0 & s+v_{d} & 0 \\
0 & 0 & 0 & 0 & s+v_{v}
\end{array}\right)
$$

and $M(\lambda, c):=\widetilde{A} \lambda^{2}-\widetilde{B} \lambda+\mathcal{F}-\mathcal{V}$. Then (3.1) can be rewritten as $M Q^{T}=0$, where $Q=\left(q_{1}, q_{2}, q_{3}, q_{4}, q_{5}\right)$. Let $A=\mathcal{V}^{-1} \widetilde{A}$ and $B=\mathcal{V}^{-1} \widetilde{B}$. Consider the equation $\left(-A \lambda^{2}+B \lambda+I\right)^{-1}\left(\mathcal{V}^{-1} \mathcal{F}\right) Q=Q$. A direct calculation yields

$$
\begin{aligned}
\bar{M}(\lambda, c):= & \left(-A \lambda^{2}+B \lambda+I\right)^{-1}\left(\mathcal{V}^{-1} \mathcal{F}\right) \\
= & \left(\begin{array}{lllll}
\frac{\alpha_{c}}{\Theta_{1}(\lambda, s)} & 0 & 0 & 0 & \frac{\beta_{c} S_{c 0}}{\Theta_{1}(\lambda, s)} \\
0 & \frac{\alpha_{e w}}{\Theta_{2}(\lambda, s)} & \frac{\alpha_{i w}}{\Theta_{2}(\lambda, s)} & 0 & \frac{\beta_{w} S_{w 0}}{\Theta_{2}(\lambda, s)} \\
0 & \frac{\alpha_{e w} \mu_{w}}{\Theta_{3}(\lambda, s)} & \frac{\alpha_{i w} \mu_{w}}{\Theta_{3}(\lambda, s)} & 0 & \frac{\beta_{w} S_{w 0} \mu_{w}}{\Theta_{3}(\lambda, s)} \\
0 & 0 & 0 & \frac{\alpha_{d}}{\Theta_{4}(\lambda, s)} & \frac{\beta_{d} N_{d}}{\Theta_{4}(\lambda, s)} \\
\frac{r_{c}}{\Theta_{5}(\lambda, s)} & \frac{r_{e w}}{\Theta_{5}(\lambda, s)} & \frac{r_{i w}}{\Theta_{5}(\lambda, s)} & \frac{r_{d}}{\Theta_{5}(\lambda, s)} & 0
\end{array}\right),
\end{aligned}
$$

where $\Theta_{1}(\lambda, s)=\lambda\left(s+v_{c}\right)+d_{i c}, \Theta_{2}(\lambda, s)=\lambda\left(s+v_{w}\right)-D_{w} \lambda^{2}+\mu_{w}, \Theta_{3}(\lambda, s)=$ $\left(\lambda s+d_{i w}\right)\left[\lambda\left(s+v_{w}\right)-D_{w} \lambda^{2}+\mu_{w}\right], \Theta_{4}(\lambda, s)=\lambda\left(s+v_{d}\right)-D_{d} \lambda^{2}+\gamma_{d}, \Theta_{5}(\lambda, s)=$ $\lambda\left(s+v_{v}\right)-D_{v} \lambda^{2}+\left(d_{v}+d_{n}\right)$. Take

$$
D=\max \left\{D_{w}, D_{d}, D_{v}\right\} \quad \text { and } \quad v_{\min }=\min \left\{0, v_{c}, v_{w}, v_{d}, v_{v}\right\} .
$$

Then for $s \geq-v_{\min }$, we have $s+v_{j} \geq s+v_{\min }$ with $j=c, w, d, v$. 
As $\Theta_{i}\left(\frac{s+v_{\min }}{2 D}, s\right)$ is increasing and nonnegative for $s \in\left[-v_{\min }, \infty\right)$, we conclude that the matrix $\bar{M}\left(\frac{s+v_{\min }}{2 D}, s\right)$ is decreasing in $s \in\left[-v_{\min },+\infty\right)$. In particular, this matrix becomes $\mathcal{V}^{-1} \mathcal{F}$ when $s=-v_{\text {min }}$ and approaches zero when $s \rightarrow+\infty$. Since $\rho(M)$ is continuous and monotonically increasing with respect to the nonnegative matrix $M$, there exists a unique $s^{*}>-v_{\min }$ such that $\rho\left(\bar{M}\left(\frac{s^{*}+v_{\min }}{2 D}, s^{*}\right)\right)=1$ and $\rho\left(\bar{M}\left(\frac{s+v_{\min }}{2 D}, s\right)\right)<1$ for $s>s^{*}$. Now we fix $s>s^{*}$. Since $\Theta_{i}(\lambda, s)$ is increasing in $\lambda \in\left[0, \frac{s+v_{\min }}{2 D}\right]$, we conclude that the matrix $\bar{M}(\lambda, s)$ is decreasing and nonnegative in $\lambda \in\left[0, \frac{s+v_{\min }}{2 D}\right]$. Consequently, there exists a $\lambda_{s} \in\left(0, \frac{s+v_{\min }}{2 D}\right)$ such that

$$
\rho(\bar{M}(\lambda, s))\left\{\begin{array}{l}
=1 \text { if } \lambda=\lambda_{s}, \\
<1 \text { if } \lambda \in\left(\lambda_{s}, \frac{s+v_{\min }}{2 D}\right], \\
>1 \text { if } \lambda \in\left[0, \lambda_{s}\right) .
\end{array}\right.
$$

Lemma 3.1. Assume that $\mathcal{R}_{0}=\rho\left(\mathcal{F} \mathcal{V}^{-1}\right)>1$. Then there exists $s^{*}>-v_{\text {min }}$ such that for each $s>s^{*}$, there exist $\lambda_{s} \in\left(0, \frac{s+v_{\min }}{2 D}\right)$ and $Q_{s}>0$ satisfying $\operatorname{det} M\left(\lambda_{s}, s\right)=0$ and $M\left(\lambda_{s}, s\right) Q_{s}=0$.

Proof. Following the above arguments, we know that $\rho\left(\bar{M}\left(\lambda_{s}, s\right)\right)=1$. Then the Perron-Frobenius theorem implies that there exists a $Q_{s} \in \mathbb{R}^{5}$ with positive components such that $\bar{M}\left(\lambda_{s}, s\right) Q_{s}=Q_{s}$. Multiplying the matrix $-A \lambda_{s}^{2}+B \lambda_{s}+I$ on the two sides of the last equality, we have $\left(A \lambda_{s}^{2}-B \lambda_{s}+\mathcal{V}^{-1} \mathcal{F}-I\right) Q_{s}=0$. Multiplying $\mathcal{V}$ to the above equality yields $M(\lambda, s) Q_{s}=0$, completing the proof.

In the sequel, we let $Q_{s}:=\left(q_{1}, q_{2}, q_{3}, q_{4}, q_{5}\right)^{T}$ as obtained in Lemma 3.1.

Lemma 3.2. The vector valued map $\Phi(x)=\left(\phi_{1}(x), \phi_{2}(x), \phi_{3}(x), \phi_{4}(x), \phi_{5}(x)\right)^{T}$ with $\phi_{i}(x)=q_{i} e^{\lambda_{s} x}$ satisfies the following system:

$$
\begin{aligned}
s \phi_{1}^{\prime}(x)= & \beta_{c} S_{c 0} \phi_{5}(x)+\alpha_{c} \phi_{1}(x)-d_{i c} \phi_{1}(x)-v_{c} \phi_{1}^{\prime}(x) \\
s \phi_{2}^{\prime}(x)= & \beta_{w} S_{w 0} \phi_{5}(x)+\alpha_{e w} \phi_{2}(x)+\alpha_{i w} \phi_{3}(x) \\
& -\mu_{w} \phi_{2}(x)-v_{w} \phi_{2}^{\prime}(x)+D_{w} \phi_{2}^{\prime \prime}(x) \\
s \phi_{3}^{\prime}(x)= & \mu_{w} \phi_{2}(x)-d_{i w} \phi_{3}(x) \\
s \phi_{4}^{\prime}(x)= & \beta_{d} N_{d} \phi_{5}(x)+\alpha_{d} \phi_{4}(x)-\gamma_{d} \phi_{4}(x)-v_{d} \phi_{4}^{\prime}(x)+D_{d} \phi_{4}^{\prime \prime}(x) \\
s \phi_{5}^{\prime}(x)= & r_{c} \phi_{1}(x)+r_{e w} \phi_{2}(x)+r_{i w} \phi_{3}(x)+r_{d} \phi_{4}(x) \\
& -\left(d_{v}+d_{n}\right) \phi_{5}(x)-v_{v} \phi_{5}^{\prime}(x)+D_{v} \phi_{5}^{\prime \prime}(x)
\end{aligned}
$$

Lemma 3.3. For each $\varpi>0$ sufficiently small and $\rho>1$ large enough, the vector valued map $P(x)=\left(p_{1}(x), p_{2}(x)\right)^{T}$ defined by

$$
p_{1}(x)=\max \left\{1-\rho e^{\varpi x}, 0\right\} S_{c 0} \quad \text { and } \quad p_{2}(x)=\max \left\{1-\rho e^{\varpi x}, 0\right\} S_{w 0}
$$

satisfies the following system of differential inequalities:

$$
\begin{gathered}
D_{c} p_{1}^{\prime \prime}(x)-\left(s+v_{c}\right) p_{1}^{\prime}(x)-\beta_{c} p_{1}(x) \phi_{5}(x)-\alpha_{c} \frac{p_{1}(x) \phi_{1}(x)}{p_{1}(x)+\phi_{1}(x)} \geq 0 \\
D_{w} p_{2}^{\prime \prime}(x)-\left(s+v_{w}\right) p_{2}^{\prime}(x)-\beta_{w} p_{2}(x) \phi_{5}(x) \\
-\alpha_{e w} \frac{p_{2}(x) \phi_{2}(x)}{p_{2}(x)+\phi_{2}(x)}-\alpha_{i w} \frac{p_{2}(x) \phi_{3}(x)}{p_{2}(x)+\phi_{3}(x)} \geq 0
\end{gathered}
$$

for any $x<X^{\prime}:=-\frac{1}{\varpi} \ln \rho$. 
Proof. When $x<X^{\prime}, 1-\rho e^{\varpi x}>0$ and $p_{1}(x)=S_{c 0}\left(1-\rho e^{\varpi x}\right)$. One has

$$
\begin{aligned}
& D_{c} p_{1}^{\prime \prime}(x)-\left(s+v_{c}\right) p_{1}^{\prime}(x)-\beta_{c} p_{1}(x) \phi_{5}(x)-\alpha_{c} \frac{p_{1}(x) \phi_{1}(x)}{p_{1}(x)+\phi_{1}(x)} \\
\geq & -\rho \varpi^{2} D_{c} S_{c 0} e^{\varpi x}+\left(s+v_{c}\right) \rho \varpi S_{c 0} e^{\varpi x}-q_{5} \beta_{c} S_{c 0}\left(1-\rho e^{\varpi x}\right) e^{\lambda_{s} x}-\alpha_{c} q_{1} e^{\lambda_{s} x} \\
\geq & {\left[\rho \varpi S_{c 0}\left(\left(s+v_{c}\right)-\varpi D_{c}\right)-\left(\alpha_{c} q_{1}+q_{5} \beta_{c} S_{c 0}\right) e^{-\left(\lambda_{s}-\varpi\right) \frac{1}{\varpi} \ln \rho}\right] e^{\varpi x} } \\
= & {\left[\rho \varpi S_{c 0}\left(\left(s+v_{c}\right)-\varpi D_{c}\right)-\left(\alpha_{c} q_{1}+q_{5} \beta_{c} S_{c 0}\right) \rho^{-\left(\lambda_{s}-\varpi\right) \frac{1}{\varpi}}\right] e^{\varpi x} . }
\end{aligned}
$$

Keeping $\rho \varpi=1$ and letting $\rho \rightarrow+\infty$, there exists $\rho>0$ and $\varpi>0$ such that $\rho \varpi S_{c 0}\left(\left(s+v_{c}\right)-\varpi D_{c}\right)-\left(\alpha_{c} q_{1}+q_{5} \beta_{c} S_{c 0}\right) \rho^{-\left(\lambda_{s}-\varpi\right) \frac{1}{\varpi}}>0$, which implies that (3.7) holds. Similarly, we can prove (3.8). This completes the proof.

Lemma 3.4. Let $\varepsilon>0$ be small enough with $\varepsilon<\frac{\varpi}{2}, \varepsilon<\frac{\lambda_{s}}{2}$ and $\lambda_{s}+\varepsilon<\frac{s+v_{\min }}{2 D}$. Then the function

$$
\Psi(x)=\left(\psi_{1}(x), \psi_{2}(x), \psi_{3}(x), \psi_{4}(x), \psi_{5}(x)\right)^{T}=Q_{s} e^{\lambda_{s} x} \max \left\{1-M e^{\varepsilon x}, 0\right\}
$$

satisfies the following inequalities:

$$
\begin{aligned}
\left(s+v_{c}\right) \psi_{1}^{\prime}(x) \leq & \beta_{c} p_{1}(x) \psi_{5}(x)+\alpha_{c} \frac{p_{1}(x) \psi_{1}(x)}{p_{1}(x)+\psi_{1}(x)}-d_{i c} \psi_{1}(x) \\
\left(s+v_{w}\right) \psi_{2}^{\prime}(x) \leq & \beta_{w} p_{2}(x) \psi_{5}(x)+\alpha_{e w} \frac{p_{2}(x) \psi_{2}(x)}{p_{2}(x)+\psi_{2}(x)+\phi_{3}(x)} \\
& +\alpha_{i w} \frac{p_{2}(x) \psi_{3}(x)}{p_{2}(x)+\phi_{2}(x)+\psi_{3}(x)}-\mu_{w} \psi_{2}(x)+D_{w} \psi_{2}^{\prime \prime}, \\
s \psi_{3}^{\prime}(x) \leq & \mu_{w} \psi_{2}(x)-d_{i w} \psi_{3}(x) \\
\left(s+v_{d}\right) \psi_{4}^{\prime}(x) \leq & \beta_{d} N_{d} \psi_{5}(x)-\beta_{d} \psi_{4}(x) \phi_{5}(x) \\
& +\alpha_{d} \frac{\left(N_{d}-\psi_{4}(x)\right) \psi_{4}(x)}{N_{d}}-\gamma_{d} \psi_{4}(x)+D_{d} \psi_{4}^{\prime \prime}, \\
\left(s+v_{v}\right) \psi_{5}^{\prime}(x) \leq & r_{c} \psi_{1}(x)+r_{e w} \psi_{2}(x)+r_{i w} \psi_{3}(x)+r_{d} \psi_{4}(x) \\
& -\left(d_{v}+d_{n}\right) \psi_{5}(x)+D_{v} \psi_{5}^{\prime \prime}
\end{aligned}
$$

for $x<X^{\prime \prime}:=-\frac{1}{\varepsilon} \ln M$, where $M>0$ is sufficiently large so that $X^{\prime \prime}<X^{\prime}$. 
Proof. When $x<X^{\prime \prime}<X^{\prime}, \psi_{i}(x)=q_{i} e^{\lambda_{s} x}\left(1-M e^{\varepsilon x}\right), p_{1}(x)=S_{c 0}\left(1-\rho e^{\varpi x}\right)$ and $p_{2}(x)=S_{w 0}\left(1-\rho e^{\varpi x}\right)$, where $i=1,2,3,4,5$. Consequently, we have

$$
\begin{aligned}
& s \psi_{1}^{\prime}(x)-\beta_{c} p_{1}(x) \psi_{5}(x)-\alpha_{c} \frac{p_{1}(x) \psi_{1}(x)}{p_{1}(x)+\psi_{1}(x)}+d_{i c} \psi_{1}(x)+v_{c} \psi_{1}^{\prime}(x) \\
= & -\left(s+v_{c}\right) q_{1}\left(\lambda_{s}+\varepsilon\right) M e^{\left(\lambda_{s}+\varepsilon\right) x}+q_{5} \beta_{c} S_{c 0} e^{\lambda_{s} x}\left(M e^{\varepsilon x}+\rho e^{\varpi x}-\rho M e^{(\varpi+\varepsilon) x}\right) \\
& +\alpha_{c} q_{1} M e^{\left(\lambda_{s}+\varepsilon\right) x}+\frac{q_{1}^{2} \alpha_{c} e^{2 \lambda_{s} x}\left(1-M e^{\varepsilon x}\right)^{2}}{S_{c 0}\left(1-\rho e^{\varpi x}\right)+q_{1} e^{\lambda_{s} x}\left(1-M e^{\varepsilon x}\right)}-q_{1} d_{i c} M e^{\left(\lambda_{s}+\varepsilon\right) x} \\
\leq & M\left[-\left(s+v_{c}\right)\left(\lambda_{s}+\varepsilon\right) q_{1}+\alpha_{c} q_{1}-d_{i c} q_{1}+q_{5} \beta_{c} S_{c 0}\right] e^{\left(\lambda_{s}+\varepsilon\right) x} \\
& +\frac{q_{1}^{2} \alpha_{c} e^{2 \lambda_{s} x}}{S_{c 0}\left(1-\rho e^{\varpi x}\right)}+\rho q_{5} \beta_{c} S_{c 0} e^{\left(\lambda_{s}+\varpi\right) x} \\
\leq & \left\{\begin{array}{l}
-\varepsilon q_{1} M+\frac{q_{1}^{2} \alpha_{c}}{S_{c 0}\left(1-\rho e^{-\varpi \frac{1}{\varepsilon} \ln M}\right)}+\rho q_{5} \beta_{c} S_{c 0} e^{-(\varpi-\varepsilon) \frac{1}{\varepsilon} \ln M}
\end{array}\right\} e^{\left(\lambda_{s}+\varepsilon\right) x} .
\end{aligned}
$$

Then for sufficiently large $M>0$, we have that (3.9) holds.

The proofs for (3.10)-(3.13) are similar and thus are omitted.

Let $X^{*}:=-\frac{1}{\varepsilon} \ln \frac{M\left(\lambda_{s}+\varepsilon\right)}{\lambda_{s}}<X^{\prime \prime}$. It is obvious that $\varphi_{i}(\cdot)$ is increasing on $\left(-\infty, X^{*}\right]$. For $X>-X^{*}$, we define

$$
\left.\Gamma=\left\{\begin{array}{l|l}
\chi_{1}(\cdot) \\
\chi_{2}(\cdot) \\
\varphi_{1}(\cdot) \\
\varphi_{2}(\cdot) \\
\varphi_{3}(\cdot) \\
\varphi_{4}(\cdot) \\
\varphi_{5}(\cdot)
\end{array}\right) \in C\left(\bar{\Omega}, \mathbb{R}^{7}\right) \mid \begin{array}{l}
p_{1}(x) \leq \chi_{1}(x) \leq S_{c 0}, p_{2}(x) \leq \chi_{2}(x) \leq S_{w 0} \\
\text { for } x \in[-X, X], \chi_{i}( \pm X)=p_{i}( \pm X), \\
\psi_{j}(x) \leq \varphi_{j}(x) \leq \phi_{j}(x) \text { for } x \in[-X, X], \\
i=1,2 \text { and } j=1,2,3,4,5 \\
\varphi_{1}(-X)=\psi_{1}(-X), \varphi_{2}( \pm X)=\psi_{2}( \pm X), \\
\varphi_{3}(-X)=\psi_{3}(-X), \varphi_{4}( \pm X)=\psi_{4}( \pm X), \\
\varphi_{5}( \pm X)=\psi_{5}( \pm X) . \\
\text { Furthermore, } \varphi_{4}(x) \leq N_{d} \text { for } x \in[-X, X] .
\end{array}\right\}
$$

where $\bar{\Omega}=[-X, X]$. For any given $\left(\chi_{1}(\cdot), \chi_{2}(\cdot), \varphi_{1}(\cdot), \varphi_{2}(\cdot), \varphi_{3}(\cdot), \varphi_{4}(\cdot), \varphi_{5}(\cdot)\right)$ $\in \Gamma$, we consider the following boundary value problems:

$$
\begin{aligned}
& -D_{c} S_{c}^{\prime \prime}(x)+\left(s+v_{c}\right) S_{c}^{\prime}(x)+\left(\beta_{c} \varphi_{5}(x)+\alpha_{c}\right) S_{c}(x)=\alpha_{c} g_{1}(x), \\
& -D_{w} S_{w}^{\prime \prime}(x)+\left(s+v_{w}\right) S_{w}^{\prime}(x)+\left(\beta_{w} \varphi_{5}(x)+\alpha_{e w}+\alpha_{i w}\right) S_{w}(x) \\
& =\alpha_{e w} g_{21}(x)+\alpha_{i w} g_{22}(x) \\
& \left(s+v_{c}\right) I_{c}^{\prime}(x)+d_{i c} I_{c}(x)=\beta_{c} \chi_{1}(x) \varphi_{5}(x)+\alpha_{c} f_{1}(x) \\
& -D_{w} E_{w}^{\prime \prime}(x)+\left(s+v_{w}\right) E_{w}^{\prime}(x)+\mu_{w} E_{w}(x) \\
& =\beta_{c} \chi_{2}(x) \varphi_{5}(x)+\alpha_{e w} f_{21}(x)+\alpha_{i w} f_{22}(x), \\
& \quad s I_{w}^{\prime}(x)+d_{i w} I_{w}(x)=\mu_{w} \varphi_{2}(x) \\
& -D_{d} I_{d}^{\prime \prime}(x)+\left(s+v_{d}\right) I_{d}^{\prime}(x)+\left(\gamma_{d}+\alpha_{d}+\beta_{d} \varphi_{5}(x)\right) I_{d}(x) \\
& =\beta_{d} N_{d} \varphi_{5}(x)+\frac{\alpha_{d}}{N_{d}}\left(2 N_{d}-\varphi_{4}(x)\right) \varphi_{4}(x) \\
& -D_{v} V^{\prime \prime}(x)+\left(s+v_{v}\right) V^{\prime}(x)+\left(d_{v}+d_{n}\right) V(x) \\
& =r_{c} \varphi_{1}(x)+r_{e w} \varphi_{2}(x)+r_{i w} \varphi_{3}(x)+r_{d} \varphi_{4}(x)
\end{aligned}
$$


with

$$
\begin{aligned}
S_{c}( \pm X) & =p_{1}( \pm X), S_{w}( \pm X)=p_{2}( \pm X), I_{c}(-X)=\psi_{1}(-X) \\
E_{w}( \pm X) & =\psi_{2}( \pm X), I_{w}(-X)=\psi_{3}(-X) \\
I_{d}( \pm X) & =\psi_{4}( \pm X), V( \pm X)=\psi_{5}( \pm X)
\end{aligned}
$$

where

$$
\begin{gathered}
f_{1}\left[\chi_{1}, \varphi_{1}\right](x)= \begin{cases}\frac{\alpha_{c} \chi_{1}(x) \varphi_{1}(x)}{\chi_{1}(x)+\varphi_{1}(x)}, & \chi_{1}(x) \varphi_{1}(x) \neq 0, \\
0, & \chi_{1}(x) \varphi_{1}(x)=0,\end{cases} \\
g_{1}\left[\chi_{1}, \varphi_{1}\right](x)= \begin{cases}\frac{\alpha_{c} \chi_{1}^{2}(x)}{\chi_{1}(x)+\varphi_{1}(x)}, & \chi_{1}(x) \neq 0, \\
0, & \chi_{1}(x)=0,\end{cases} \\
f_{21}\left[\chi_{2}, \varphi_{2}, \varphi_{3}\right](x)= \begin{cases}\frac{\alpha_{e w} \chi_{2}(x) \varphi_{2}(x)}{\chi_{2}(x)+\varphi_{2}(x)+\varphi_{3}(x)}, & \chi_{2}(x) \varphi_{2}(x) \neq 0, \\
0, & \chi_{2}(x) \varphi_{2}(x)=0,\end{cases} \\
f_{22}\left[\chi_{2}, \varphi_{2}, \varphi_{3}\right](x)= \begin{cases}\frac{\alpha_{i w} \chi_{2}(x) \varphi_{3}(x)}{\chi_{2}(x)+\varphi_{2}(x)+\varphi_{3}(x)}, & \chi_{2}(x) \varphi_{3}(x) \neq 0, \\
0, & \chi_{2}(x) \varphi_{3}(x)=0,\end{cases} \\
g_{21}\left[\chi_{2}, \varphi_{2}, \varphi_{3}\right](x)= \begin{cases}\frac{\alpha_{e w} \chi_{2}(x)\left(\chi_{2}(x)+\varphi_{3}(x)\right)}{\chi_{2}(x)+\varphi_{2}(x)+\varphi_{3}(x)}, & \chi_{2}(x)\left(\chi_{2}(x)+\varphi_{3}(x)\right) \neq 0, \\
0, & \chi_{2}(x)\left(\chi_{2}(x)+\varphi_{3}(x)\right)=0,\end{cases} \\
g_{22}\left[\chi_{2}, \varphi_{2}, \varphi_{3}\right](x)= \begin{cases}\frac{\alpha_{i w} \chi_{2}(x)\left(\chi_{2}(x)+\varphi_{2}(x)\right)}{\chi_{2}(x)+\varphi_{2}(x)+\varphi_{3}(x)}, & \chi_{2}(x)\left(\chi_{2}(x)+\varphi_{2}(x)\right) \neq 0, \\
0, & \chi_{2}(x)\left(\chi_{2}(x)+\varphi_{2}(x)\right)=0 .\end{cases}
\end{gathered}
$$

It is not difficult to verify that $f_{1}(x), f_{21}(x), f_{22}(x), g_{1}(x), g_{21}(x), g_{22}(x)$ are continuous functions of $x \in[-X, X]$. Then the problems (3.14)-(3.20) and (3.21) admit a unique solution $\left(S_{c}(\cdot), S_{w}(\cdot), I_{c}(\cdot), E_{w}(\cdot), I_{w}(\cdot), I_{d}(\cdot), V(\cdot)\right)$ with $I_{c}, I_{w}$ $\in C^{1}[-X, X], E_{w}, V \in C^{2}[-X, X]$ and $S_{c}, S_{w}, I_{d} \in W^{2, p}(-X, X)$, where $p \geq 2$. (In fact, (3.14), (3.15) and (3.19) hold for almost everywhere $x \in(-X, X)$; see [1, 2, 6].) This then gives an operator $T=\left(T_{1}, T_{2}, T_{3}, T_{4}, T_{5}, T_{6}, T_{7}\right)$ defined on $\Gamma$ as

$$
\begin{aligned}
S_{c} & =T_{1}\left(\chi_{1}, \chi_{2}, \varphi_{1}, \varphi_{2}, \varphi_{3}, \varphi_{4}, \varphi_{5}\right), S_{w}=T_{2}\left(\chi_{1}, \chi_{2}, \varphi_{1}, \varphi_{2}, \varphi_{3}, \varphi_{4}, \varphi_{5}\right), \\
I_{c} & =T_{3}\left(\chi_{1}, \chi_{2}, \varphi_{1}, \varphi_{2}, \varphi_{3}, \varphi_{4}, \varphi_{5}\right), E_{w}=T_{4}\left(\chi_{1}, \chi_{2}, \varphi_{1}, \varphi_{2}, \varphi_{3}, \varphi_{4}, \varphi_{5}\right), \\
I_{w} & =T_{5}\left(\chi_{1}, \chi_{2}, \varphi_{1}, \varphi_{2}, \varphi_{3}, \varphi_{4}, \varphi_{5}\right), I_{d}=T_{6}\left(\chi_{1}, \chi_{2}, \varphi_{1}, \varphi_{2}, \varphi_{3}, \varphi_{4}, \varphi_{5}\right), \\
V & =T_{7}\left(\chi_{1}, \chi_{2}, \varphi_{1}, \varphi_{2}, \varphi_{3}, \varphi_{4}, \varphi_{5}\right) .
\end{aligned}
$$

Theorem 3.5. The operator $T$ maps $\Gamma$ into $\Gamma$.

Proof. Let $\left(\chi_{1}(\cdot), \chi_{2}(\cdot), \varphi_{1}(\cdot), \varphi_{2}(\cdot), \varphi_{3}(\cdot), \varphi_{4}(\cdot), \varphi_{5}(\cdot)\right) \in \Gamma$ and

$$
\left(S_{c}(\cdot), S_{w}(\cdot), I_{c}(\cdot), E_{w}(\cdot), I_{w}(\cdot), I_{d}(\cdot), V(\cdot)\right)=T\left(\chi_{1}, \chi_{2}, \varphi_{1}, \varphi_{2}, \varphi_{3}, \varphi_{4}, \varphi_{5}\right)(\cdot) .
$$

By virtue of the embedding theorem, we have $S_{c}(\cdot), S_{w}(\cdot), I_{d}(\cdot) \in C([-X, X], \mathbb{R})$. Now we show that $p_{2}(x) \leq S_{w}(x) \leq S_{w 0}$ for $x \in[-X, X]$. Since 0 is a subsolution of (3.15), by the maximum principle [6] we have $S_{w}(x) \geq 0$ for $x \in[-X, X]$. Furthermore, since $\left(\beta_{w} \varphi_{5}(x)+\alpha_{e w}+\alpha_{i w}\right) S_{w 0} \geq \alpha_{e w} g_{21}(x)+\alpha_{i w} g_{22}(x)$ for $x \in$ $(-X, X)$ and $S_{w}( \pm X) \leq S_{w 0}, S_{w 0}$ is a supersolution of (3.15), and hence we have 
$S_{w}(x) \leq S_{w 0}$ for $x \in[-X, X]$. Note that $S_{w}(-X)=p_{2}(-X)$ and $S_{w}\left(X^{\prime}\right) \geq$ $p_{2}\left(X^{\prime}\right)=0$. Then for $x \in\left(-X, X^{\prime}\right)$, by (3.8) we have

$$
\begin{aligned}
0 \geq & -D_{w} p_{2}^{\prime \prime}(x)+\left(s+v_{w}\right) p_{2}^{\prime}(x)+\beta_{w} \phi_{5}(x) p_{2}(x) \\
& +\alpha_{e w} \frac{p_{2}(x) \phi_{2}(x)}{p_{2}(x)+\phi_{2}(x)}+\alpha_{i w} \frac{p_{2}(x) \phi_{3}(x)}{p_{2}(x)+\phi_{3}(x)} \\
\geq & -D_{w} p_{2}^{\prime \prime}(x)+\left(s+v_{w}\right) p_{2}^{\prime}(x)+\beta_{w} \varphi_{5}(x) p_{2}(x) \\
& +\alpha_{e w} \frac{p_{2}(x) \varphi_{2}(x)}{p_{2}(x)+\varphi_{2}(x)+\varphi_{3}(x)}+\alpha_{i w} \frac{p_{2}(x) \varphi_{3}(x)}{p_{2}(x)+\varphi_{2}(x)+\varphi_{3}(x)} \\
= & -D_{w} p_{2}^{\prime \prime}(x)+\left(s+v_{w}\right) p_{2}^{\prime}(x)+\left(\beta_{w} \varphi_{5}(x)+\alpha_{e w}+\alpha_{i w}\right) p_{2}(x) \\
& -\alpha_{e w} \frac{p_{2}(x)\left(p_{2}(x)+\varphi_{3}(x)\right)}{p_{2}(x)+\varphi_{2}(x)+\varphi_{3}(x)}-\alpha_{i w} \frac{p_{2}(x)\left(p_{2}(x)+\varphi_{2}(x)\right)}{p_{2}(x)+\varphi_{2}(x)+\varphi_{3}(x)} \\
\geq & -D_{w} p_{2}^{\prime \prime}(x)+\left(s+v_{w}\right) p_{2}^{\prime}(x)+\left(\beta_{w} \phi_{5}(x)+\alpha_{e w}+\alpha_{i w}\right) p_{2}(x) \\
& -\alpha_{e w} g_{21}(x)-\alpha_{i w} g_{22}(x),
\end{aligned}
$$

which implies that $p_{2}(\cdot)$ is a subsolution of (3.15) on $\left[-X, X^{\prime}\right]$. Here we used the fact that the function $\frac{u(u+a)}{u+v+a}$ is nondecreasing on $u$ and nonincreasing on $v$ for $(u, v) \in(0,+\infty) \times[0,+\infty)$, where $a \geq 0$. Consequently, the maximum principle yields that $S_{w}(x) \geq p_{2}(x)$ for $x \in\left[-X, X^{\prime}\right]$. Combining the above arguments, we have $p_{2}(x) \leq S_{w}(x) \leq S_{w 0}$ for $x \in[-X, X]$. Similarly, we can confirm that $p_{1}(x) \leq S_{c}(x) \leq S_{c 0}$ for $x \in[-X, X]$.

We now show that $\psi_{2}(x) \leq E_{w}(x) \leq \phi_{2}(x)$ for $x \in[-X, X]$. By (3.3), we have

$$
\begin{aligned}
& -D_{w} \phi_{2}^{\prime \prime}(x)+\left(s+v_{w}\right) \phi_{2}^{\prime}(x)+\mu_{w} \phi_{2}(x) \\
= & \beta_{w} S_{w 0} \phi_{5}(x)+\alpha_{e w} \phi_{2}(x)+\alpha_{i w} \phi_{3}(x) \\
\geq & \beta_{c} \chi_{2}(x) \varphi_{5}(x)+\frac{\alpha_{e w} \chi_{2}(x) \phi_{2}(x)}{\phi_{2}(x)+\chi_{2}(x)}+\frac{\alpha_{e w} \chi_{2}(x) \phi_{3}(x)}{\phi_{3}(x)+\chi_{2}(x)} \\
\geq & \beta_{c} \chi_{2}(x) \varphi_{5}(x)+\alpha_{e w} f_{21}(x)+\alpha_{i w} f_{22}(x) .
\end{aligned}
$$

This, combined with $\psi_{2}( \pm X)=E_{w}( \pm X) \leq \phi_{2}( \pm X)$, implies that $\phi_{2}(\cdot)$ is a supersolution of (3.17) on $[-X, X]$. The maximum principle implies that $E_{w}(x) \leq$ $\phi_{2}(x)$ for $x \in[-X, X]$. Furthermore, since 0 is a subsolution of (3.17), we have that $E_{w}(x) \geq 0$ for $x \in[-X, X]$. Then for $x \in\left(-X, X^{\prime \prime}\right)$, by (3.10) we have

$$
\begin{aligned}
& -D_{w} \psi_{2}^{\prime \prime}+\left(s+v_{w}\right) \psi_{2}^{\prime}(x)+\mu_{w} \psi_{2}(x) \\
\leq & \beta_{w} p_{2}(x) \psi_{5}(x)+\alpha_{e w} \frac{p_{2}(x) \psi_{2}(x)}{p_{2}(x)+\psi_{2}(x)+\phi_{3}(x)}+\alpha_{i w} \frac{p_{2}(x) \psi_{3}(x)}{p_{2}(x)+\phi_{2}(x)+\psi_{3}(x)} \\
\leq & \beta_{w} \chi_{2}(x) \varphi_{5}(x)+\alpha_{e w} \frac{\chi_{2}(x) \varphi_{2}(x)}{\chi_{2}(x)+\varphi_{2}(x)+\varphi_{3}(x)}+\alpha_{i w} \frac{\chi_{2}(x) \varphi_{3}(x)}{\chi_{2}(x)+\varphi_{2}(x)+\varphi_{3}(x)} \\
= & \beta_{c} \chi_{2}(x) \varphi_{5}(x)+\alpha_{e w} f_{21}(x)+\alpha_{i w} f_{22}(x) .
\end{aligned}
$$

In view of $\psi_{2}(-X)=E_{w}(-X)$ and $0 \leq \psi_{2}\left(X^{\prime \prime}\right) \leq E_{w}\left(X^{\prime \prime}\right)$, an application of the maximum principle yields $E_{w}(x) \geq \psi_{2}(x)$ for $x \in\left[-X, X^{\prime \prime}\right]$. Thus, we have proved that $\psi_{2}(x) \leq E_{w}(x) \leq \phi_{2}(x)$ for $x \in[-X, X]$. Similarly, we can prove that $\psi_{1}(x) \leq I_{c}(x) \leq \phi_{1}(x), \psi_{3}(x) \leq I_{w}(x) \leq \phi_{3}(x)$ and $\psi_{5}(x) \leq V(x) \leq \phi_{5}(x)$ for $x \in[-X, X]$. 
Finally, we prove that $\psi_{4}(x) \leq I_{d}(x) \leq \min \left\{\phi_{4}(x), N_{d}\right\}$ for any $x \in[-X, X]$. Note that $2 N_{d} x-x^{2}$ is increasing on $x \in\left(0, N_{d}\right)$. Then we have

$$
\left(\gamma_{d}+\alpha_{d}+\beta_{d} \varphi_{5}(x)\right) N_{d}>\beta_{d} N_{d} \varphi_{5}(x)+\frac{\alpha_{d}}{N_{d}}\left(2 N_{d}-\varphi_{4}(x)\right) \varphi_{4}(x),
$$

which implies that $N_{d}$ is a supersolution of (3.19) on $x \in[-X, X]$. Consequently, we have $I_{d}(x) \leq N_{d}$ for $x \in[-X, X]$. Similarly, we have $I_{d}(x) \geq 0$ for $x \in[-X, X]$. Furthermore, by (3.12) we have

$$
\begin{aligned}
& -D_{d} \psi_{4}^{\prime \prime}+\left(s+v_{d}\right) \psi_{4}^{\prime}(x)+\left(\gamma_{d}+\alpha_{d}+\beta_{d} \varphi_{5}(x)\right) \psi_{4}(x) \\
& \quad \leq-D_{d} \psi_{4}^{\prime \prime}+\left(s+v_{d}\right) \psi_{4}^{\prime}(x)+\left(\gamma_{d}+\alpha_{d}+\beta_{d} \phi_{5}(x)\right) \psi_{4}(x) \\
& \leq \beta_{d} N_{d} \psi_{5}(x)+\alpha_{d} \frac{\left(2 N_{d}-\psi_{4}(x)\right) \psi_{4}(x)}{N_{d}} \\
& \quad \leq \beta_{d} N_{d} \varphi_{5}(x)+\alpha_{d} \frac{\left(2 N_{d}-\varphi_{4}(x)\right) \varphi_{4}(x)}{N_{d}},
\end{aligned}
$$

which implies that $\psi_{4}(x)$ is a subsolution of (3.19) on $\left[-X, X^{\prime \prime}\right]$. Therefore, we have $I_{d}(x) \geq \psi_{4}(x)$ for $x \in\left[-X, X^{\prime \prime}\right]$. On the other hand, inequality (3.5) implies that $\phi_{4}$ is a supersolution of (3.19), and hence $I_{d}(x) \leq \phi_{4}(x)$ for $x \in[-X, X]$.

By the classical embedding theorems, we have that $T$ is a compact operator from $\Gamma$ into $\Gamma$. Now we show that $T: \Gamma \rightarrow \Gamma$ is continuous. First, we show that $f_{1}, f_{21}, f_{22}, g_{1}, g_{21}, g_{22}$ are continuous operators from $\Gamma$ to $C\left([-X, X], \mathbb{R}_{+}\right)$. Consider $f_{1}$ first. Let $\chi_{1}^{1}, \chi_{1}^{2}, \varphi_{1}^{1}, \varphi_{1}^{2} \in C[-X, X]$ with $p_{1}(x) \leq \chi_{1}^{1}(x), \chi_{1}^{2}(x) \leq S_{c 0}$ and $\psi_{1}(x) \leq \varphi_{1}^{1}(x), \varphi_{1}^{2}(x) \leq \phi_{1}(x)$ for $x \in[-X, X]$. When $\chi_{1}^{1}(x) \varphi_{1}^{1}(x) \chi_{1}^{2}(x) \varphi_{1}^{2}(x) \neq$ 0 , we have

$$
\begin{aligned}
& \left|f_{1}\left[\chi_{1}^{1}, \varphi_{1}^{1}\right](x)-f_{1}\left[\chi_{1}^{2}, \varphi_{1}^{2}\right](x)\right| \\
\leq & \frac{\alpha_{c} \chi_{1}^{1}(x) \chi_{1}^{2}(x)\left|\varphi_{1}^{1}(x)-\varphi_{1}^{2}(x)\right|}{\left[\chi_{1}^{1}(x)+\varphi_{1}^{1}(x)\right]\left[\chi_{1}^{2}(x)+\varphi_{1}^{2}(x)\right]}+\frac{\alpha_{c} \varphi_{1}^{1}(x) \varphi_{1}^{2}(x)\left|\chi_{1}^{1}(x)-\chi_{1}^{2}(x)\right|}{\left[\chi_{1}^{1}(x)+\varphi_{1}^{1}(x)\right]\left[\chi_{1}^{2}(x)+\varphi_{1}^{2}(x)\right]} \\
\leq & \alpha_{c}\left|\varphi_{1}^{1}(x)-\varphi_{1}^{2}(x)\right|+\alpha_{c}\left|\chi_{1}^{1}(x)-\chi_{1}^{2}(x)\right| .
\end{aligned}
$$

When $\chi_{1}^{1}(x) \varphi_{1}^{1}(x) \chi_{1}^{2}(x) \varphi_{1}^{2}(x)=0$ and $\chi_{1}^{1}(x) \varphi_{1}^{1}(x)+\chi_{1}^{2}(x) \varphi_{1}^{2}(x) \neq 0$, for example, $\chi_{1}^{1}(x)=0$ and $\chi_{1}^{2}(x) \varphi_{1}^{2}(x) \neq 0$, we have

$$
\begin{aligned}
\left|f_{1}\left[\chi_{1}^{1}, \varphi_{1}^{1}\right](x)-f_{1}\left[\chi_{1}^{2}, \varphi_{1}^{2}\right](x)\right| & =\left|\frac{\alpha_{c} \varphi_{1}^{2}(x)}{\chi_{1}^{2}(x)+\varphi_{1}^{2}(x)}\right|\left|\chi_{1}^{1}(x)-\chi_{1}^{2}(x)\right| \\
& \leq \alpha_{c}\left|\chi_{1}^{1}(x)-\chi_{1}^{2}(x)\right| .
\end{aligned}
$$

Therefore, $f_{1}$ is continuous. Similarly, we can prove that $f_{21}, f_{22}, g_{1}, g_{21}, g_{22}$ are continuous. Consequently, using the continuous dependence of solutions of ODEs on initial values and the standard elliptic estimates [1, 2, 6, we have that $T_{3}, T_{4}, T_{5}, T_{7}$ are continuous operators on $\Gamma$. Furthermore, let $S_{c}^{i}=T_{1}\left(\chi_{1}^{i}, \chi_{2}^{i}, \varphi_{1}^{i}, \varphi_{2}^{i}, \varphi_{3}^{i}, \varphi_{4}^{i}, \varphi_{5}^{i}\right)$, $i=1,2$, where $\left(\chi_{1}^{i}, \chi_{2}^{i}, \varphi_{1}^{i}, \varphi_{2}^{i}, \varphi_{3}^{i}, \varphi_{4}^{i}, \varphi_{5}^{i}\right) \in \Gamma$. Then, we have

$$
\begin{aligned}
& -D_{c}\left(S_{c}^{1}-S_{c}^{2}\right)^{\prime \prime}(x)+\left(s+v_{c}\right)\left(S_{c}^{1}-S_{c}^{2}\right)^{\prime}(x)+\left(\alpha_{c}+\beta_{c} \varphi_{5}^{1}(x)\right)\left(S_{c}^{1}-S_{c}^{2}\right)(x) \\
& \quad=\alpha_{c}\left[g_{1}\left[\chi_{1}^{1}, \varphi_{1}^{1}\right](x)-g_{1}\left[\chi_{1}^{2}, \varphi_{1}^{2}\right](x)\right]+\beta_{c} S_{c}^{2}(x)\left[\varphi_{5}^{1}(x)-\varphi_{5}^{2}(x)\right] .
\end{aligned}
$$

Note that $0 \leq S_{c}^{2}(x) \leq S_{c 0}$ for $x \in[-X, X]$. Applying the standard elliptic estimates to the last equality yields that $T_{1}$ is a continuous operator on $\Gamma$. Similarly, $T_{2}$ and $T_{6}$ are also continuous operators on $\Gamma$. 
Combining the above arguments, we know that $T: \Gamma \rightarrow \Gamma$ is a completely continuous operator. Hence, Schauder's fixed point theorem implies that there exists $\left(S_{c, X}, S_{w, X}, I_{c, X}, E_{w, X}, I_{w, X}, I_{d, X} V_{X}\right) \in \Gamma$ such that

$$
\begin{aligned}
& \left(S_{c, X}(x), S_{w, X}(x), I_{c, X}(x), E_{w, X}(x), I_{w, X}(x), I_{d, X}(x), V_{X}(x)\right) \\
& \quad=T\left(S_{c, X}, S_{w, X}, I_{c, X}, E_{w, X}, I_{w, X}, I_{d, X}, V_{X}\right)(x)
\end{aligned}
$$

for $x \in[-X, X]$.

Theorem 3.6. (i) $I_{c, X}, I_{w, X} \in C^{2}[-X, X], S_{c, X}, S_{w, X}, E_{w, X}, I_{d, X}, V_{X} \in C^{3}[-X, X]$.

(ii) There exists $M_{0}>0$ such that for any $X>-X^{*},\left\|I_{c, X}\right\|_{C^{1}(\bar{\Omega})},\left\|I_{w, X}\right\|_{C^{2}(\bar{\Omega})}$, $\left\|V_{X}\right\|_{C(\bar{\Omega})},\left\|S_{c, X}\right\|_{C^{2}(\bar{\Omega})},\left\|S_{w, X}\right\|_{C^{2}(\bar{\Omega})},\left\|E_{w, X}\right\|_{C^{2}(\bar{\Omega})} \leq M_{0}$, where $\bar{\Omega}=[-X, X]$.

(iii) For each $Y>-X^{*}$, there exists $C_{0}=C_{0}(Y)>0$ such that

$\left\|I_{c, X}^{\prime \prime}\right\|_{C(\bar{U})},\left\|S_{c, X}^{\prime \prime \prime}\right\|_{C(\bar{U})},\left\|S_{w, X}^{\prime \prime \prime}\right\|_{C(\bar{U})},\left\|E_{w, X}^{\prime \prime \prime}\right\|_{C(\bar{U})},\left\|I_{d, X}\right\|_{C^{3}(\bar{U})},\left\|V_{X}\right\|_{C^{3}(\bar{U})} \leq C_{0}$

for any $X>Y$, where $\bar{U}=[-Y, Y]$.

Proof. (i) First, we have $I_{c, X}, I_{w, X} \in C^{1}[-X, X], E_{w, X}, V_{X} \in C^{2}[-X, X]$ and $S_{c, X}, S_{w, X}, I_{d, X} \in W^{2, p}(-X, X), p \geq 2$. Then by the embedding theorem, we have $S_{c, X}, S_{w, X}, I_{d, X} \in W^{2, p}(-X, X) \hookrightarrow C^{1+\alpha}[-X, X]$ for some $\alpha \in(0,1)$. Furthermore, it is not difficult to prove that $g_{1}\left[S_{c, X}, I_{c, X}\right](\cdot), g_{21}\left[S_{w, X}, E_{w, X}, I_{w, X}\right](\cdot)$, $g_{22}\left[S_{w, X}, E_{w, X}, I_{w, X}\right](\cdot) \in C^{\alpha}[-X, X]$, which further yields $S_{c, X}, S_{w, X}, I_{d, X} \in$ $C^{2, \alpha}[-X, X]$. Then, we can verify that the operators $f_{1}, f_{21}, f_{22}, g_{1}, g_{21}$ and $g_{22}$ map the solutions $\left(S_{c, X}, S_{w, X}, I_{c, X}, E_{w, X}, I_{w, X}, I_{d, X}, V_{X}\right)$ into $C^{1}[-X, X]$. Therefore, $I_{c, X}, I_{w, X} \in C^{2}[-X, X], S_{c, X}, S_{w, X}, E_{w, X}, I_{d, X}, V_{X} \in C^{3}[-X, X]$.

(ii) It is obvious that $\left(S_{c, X}, S_{w, X}, I_{c, X}, E_{w, X}, I_{w, X}, I_{d, X}, V_{X}\right) \in \Gamma$ satisfies

$$
\begin{aligned}
\left(s+v_{c}\right) S_{c, X}^{\prime}= & -\beta_{c} S_{c, X} V_{X}-\alpha_{c} S_{c, X} I_{c, X} / N_{c, X}+D_{c} S_{c, X}^{\prime \prime} \\
\left(s+v_{w}\right) S_{w, X}^{\prime}= & -\beta_{w} S_{w, X} V_{X}-\alpha_{e w} S_{w, X} E_{w, X} / N_{w, X} \\
& -\alpha_{i w} S_{w, X} I_{w, X} / N_{w, X}+D_{w} S_{w, X}^{\prime \prime} \\
\left(s+v_{c}\right) I_{c, X}^{\prime}= & \beta_{c} S_{c, X} V_{X}+\alpha_{c} S_{c, X} I_{c, X} / N_{c, X}-d_{i c} I_{c, X}, \\
\left(s+v_{w}\right) E_{w, X}^{\prime}= & \beta_{w} S_{w, X} V_{X}+S_{w, X}\left[\alpha_{e w} E_{w, X}+\alpha_{i w} I_{w, X}\right] / N_{w, X} \\
& -\mu_{w} E_{w, X}+D_{w} E_{w, X}^{\prime \prime} \\
s I_{w, X}^{\prime}= & \mu_{w} E_{w, X}-d_{i w} I_{w, X} \\
\left(s+v_{d}\right) I_{d, X}^{\prime}= & \beta_{d}\left(N_{d}-I_{d, X}\right) V_{X}+\alpha_{d}\left(N_{d}-I_{d, X}\right) I_{d, X} / N_{d} \\
& -\gamma_{d} I_{d, X}+D_{d} I_{d, X}^{\prime \prime} \\
\left(s+v_{v}\right) V_{X}^{\prime}= & r_{c} I_{c, X}+r_{e w} E_{w, X}+r_{i w} I_{w, X}+r_{d} I_{d, X} \\
& -\left(d_{v}+d_{n}\right) V_{X}+D_{v} V_{X}^{\prime \prime},
\end{aligned}
$$

where $N_{c, X}=S_{c, X}+I_{c, X}$ and $N_{w, X}=S_{w, X}+E_{w, X}+I_{w, X}$.

Following (3.23), we have

$$
\left[e^{-\frac{s+v_{c}}{D_{c}} x} S_{c, X}^{\prime}(x)\right]^{\prime}=\frac{1}{D_{c}} e^{-\frac{s+v_{c}}{D_{c}} x}\left[\beta_{c} S_{c, X}(x) V_{X}(x)+\alpha_{c} \frac{S_{c, X}(x) I_{c, X}(x)}{N_{c, X}(x)}\right] .
$$


Therefore, for $x \in[-X, X)$ we have

$$
\begin{aligned}
& S_{c, X}^{\prime}(x)-e^{\frac{s+v_{c}}{D_{c}}(x-X)} S_{c, X}^{\prime}(X) \\
= & -\frac{1}{D_{c}} \int_{x}^{X} e^{\frac{s+v_{c}}{D_{c}}(x-z)}\left[\beta_{c} S_{c, X}(z) V_{X}(z)+\alpha_{c} S_{c, X}(z) I_{c, X}(z) / N_{c, X}(z)\right] d z .
\end{aligned}
$$

Since $S_{c, X}^{\prime}(X) \leq 0$, we have $S_{c, X}^{\prime}(x) \leq 0$ for any $x \in[-X, X]$. From (3.23) and (3.25), we further have $\left(s+v_{c}\right)\left[S_{c, X}^{\prime}(x)+I_{c, X}^{\prime}(x)\right]+d_{i c} I_{c, X}(x)=D_{c} S_{c, X}^{\prime \prime}(x)$. Integrating two sides of the above equality from $-X$ to $X$, we have

$$
\begin{aligned}
& D_{c}\left[S_{c, X}^{\prime}(X)-S_{c, X}^{\prime}(-X)\right]-\left(s+v_{c}\right)\left[S_{c, X}(X)-S_{c, X}(-X)\right] \\
= & \left(s+v_{c}\right)\left[I_{c, X}(X)-I_{c, X}(-X)\right]+d_{i c} \int_{-X}^{X} I_{c, X}(x) d x .
\end{aligned}
$$

Since $S_{c, X}(X)=0, I_{c, X}(-X)=\psi_{1}(-X)$ and $S_{c, X}^{\prime}(-X) \geq p_{1}^{\prime}(-X)$, we have

$$
\int_{-X}^{X} I_{c, X}(x) d x \leq \frac{1}{d_{i c}}\left[-D_{c} p_{1}^{\prime}(-X)+\left(s+v_{c}\right) \psi_{1}(-X)+\left(s+v_{c}\right) S_{c 0}\right] \leq M_{0}
$$

and $I_{c, X}(X) \leq M_{0}$ for some $M_{0}>0$, which is independent of $X>-X^{*}$. Consequently, from (3.25) we get

$$
\begin{aligned}
\int_{-X}^{X} & {\left[\beta_{c} S_{c, X}(z) V_{X}(z)+\alpha_{c} S_{c, X}(z) I_{c, X}(z) / N_{c, X}(z)\right] d z } \\
& \leq\left(s+v_{c}\right)\left[I_{c, X}(X)-\psi_{1}(-X)\right]+d_{i c} \int_{-X}^{X} I_{c, X}(x) d x \leq M_{0}
\end{aligned}
$$

for some $M_{0}>0$, which is independent of $X>-X^{*}$. Integrating two sides of (3.23) from $-X$ to $x$, we then have

$$
\begin{aligned}
D_{c} S_{c, X}^{\prime}(x)= & D_{c} S_{c, X}^{\prime}(-X)+\left(s+v_{c}\right)\left[S_{c, X}(x)-S_{c, X}(-X)\right] \\
& +\int_{-X}^{X}\left[\beta_{c} S_{c, X}(z) V_{X}(z)+\alpha_{c} S_{c, X}(z) I_{c, X}(z) / N_{c, X}(z)\right] d z \\
\geq & D_{c} p_{1}^{\prime}(-X)-\left(s+v_{c}\right) S_{c 0} .
\end{aligned}
$$

Therefore, there exists $M_{0}>0$ independent of $X>-X^{*}$ such that $\left|S_{c, X}^{\prime}(x)\right| \leq M_{0}$ for $x \in[-X, X]$. Similarly, integrating two sides of (3.25) from $-X$ to $x$, we can find an $M_{0}>0$ independent of $X>-X^{*}$ such that $\left|I_{c, X}(x)\right| \leq M_{0}$ for $x \in[-X, X]$.

Consider $S_{w, X}, E_{w, X}$ and $I_{w, X}$. From (3.24) and (3.26) we have

$$
D_{w}\left[S_{w, X}(x)+E_{w, X}(x)\right]^{\prime \prime}-\left(s+v_{w}\right)\left[S_{w, X}(x)+E_{w, X}(x)\right]^{\prime}=\mu_{w} E_{w, X}(x) .
$$

Since $S_{w, X}^{\prime}(X) \leq 0$ and $E_{w, X}^{\prime}(X) \leq 0$, we can obtain that $S_{w, X}^{\prime}(x)+E_{w, X}^{\prime}(x) \leq 0$ for $x \in[-X, X]$, and hence there exists an $M_{0}>0$ independent of $X>-X^{*}$ such that $S_{w, X}(x)+E_{w, X}(x) \leq M_{0}$ for $x \in[-X, X]$. Therefore, $E_{w, X}(x) \leq M_{0}$ for $x \in[-X, X]$. Integrating (3.30) from $-X$ to $X$, we get

$$
\begin{aligned}
\int_{-X}^{X} E_{w, X}(x) d x & \leq-D_{w} S_{w, X}^{\prime}(-X)+\left(s+v_{w}\right)\left[S_{w, X}(-X)+E_{w, X}(-X)\right] \\
& \leq-D_{w} p_{2}^{\prime}(-X)+\left(s+v_{w}\right)\left[p_{2}(-X)+\psi_{2}(-X)\right] \leq M_{0}
\end{aligned}
$$

for some $M_{0}>0$ independent of $X>-X^{*}$. In the last inequalities, we used the fact that $S_{w, X}^{\prime}(X) \leq 0, E_{w, X}^{\prime}(-X) \geq 0$ and $E_{w, X}^{\prime}(X) \leq 0$. Consequently, by some 
arguments as done for $S_{c, X}$ and $I_{c, X}$, it follows from (3.24), (3.26) and (3.27) that there exists an $M_{0}>0$, which is independent of $X>-X^{*}$, such that

$$
\left\|S_{w, X}\right\|_{C^{1}[-X, X]} \leq M_{0},\left\|E_{w, X}\right\|_{C^{1}[-X, X]} \leq M_{0},\left\|I_{w, X}\right\|_{C^{2}[-X, X]} \leq M_{0} .
$$

It is easy to see that there exists an $M_{0}>\sup _{x \in \mathbb{R}} \psi_{5}(x)$ such that $M_{0}$ is a supersolution of (3.29). Then we have $V_{X}(x) \leq M_{0}$ for $x \in[-X, X]$, proving (ii).

(iii) For $Y \in\left(-X^{\prime \prime}, X\right)$, applying the $L^{p}(p \geq 2)$ estimates of linear elliptic differential equations to $V_{X}$ and (3.29), we have

$$
\begin{gathered}
\left\|V_{X}\right\|_{W^{2, p}(U)} \leq C\left(r_{c}\left\|I_{c, X}\right\|_{L^{p}(U)}+r_{e w}\left\|E_{w, X}\right\|_{L^{p}(U)}+r_{i w}\left\|I_{w, X}\right\|_{L^{p}(U)}\right. \\
\left.+r_{d}\left\|I_{d, X}\right\|_{L^{p}(U)}+\|\varphi\|_{W^{2, p}(U)}+\left\|V_{X}\right\|_{L^{p}(U)}\right)
\end{gathered}
$$

where $U=(-Y, Y), C=C(Y)>0$ is a constant and $\varphi$ is taken to be a linear function connecting the points $\left(-Y, V_{X}(-Y)\right)$ and $\left(Y, V_{X}(Y)\right)$. Consequently, we can find a constant $C_{0}>0$ which is only dependent of $Y$ such that $\left\|V_{X}\right\|_{W^{2, p}(-Y, Y)} \leq C_{0}$ for any $X \geq Y$. Since $W^{2, p}(-Y, Y) \hookrightarrow C^{1, \alpha}[-Y, Y]$ for $\alpha=1-\frac{1}{p}$, the embedding theorem further implies that there exists a constant $C>0$ only dependent on $Y$ such that $\left\|V_{X}\right\|_{C^{1, \alpha}[-Y, Y]} \leq C\left\|V_{X}\right\|_{W^{2, p}(-Y, Y)}$. Therefore, $\left\|V_{X}\right\|_{C^{1, \alpha}[-Y, Y]} \leq C_{0}$ for some $C_{0}>0$, which is only dependent on $Y$. From (3.29), we further have $\left\|V_{X}\right\|_{C^{2}[-Y, Y]} \leq C_{0}$ for some $C_{0}=C_{0}(Y)>0$. By a similar argument, we have $\left\|I_{d, X}\right\|_{C^{2}[-Y, Y]} \leq C_{0}$ for some $C_{0}=C_{0}(Y)>0$.

Finally, differentiating two sides of (3.23) $-(\underline{3.29})$ gives $(\underline{3.22})$ for some $C_{0}(Y)$.

We now establish our main results. Let $\left\{X_{n}\right\}$ be an increasing sequence with $X_{n}>X^{*}$ and $\lim _{n \rightarrow \infty} X_{n}=+\infty$. Then the solutions

$$
\left(S_{c, X_{n}}, S_{w, X_{n}}, I_{c, X_{n}}, E_{w, X_{n}}, I_{w, X_{n}}, I_{d, X_{n}}, V_{X_{n}}\right) \in \Gamma_{X_{n}}
$$

satisfy Theorem 3.6 as well as (3.23)-(3.29). We can assume (if necessary, taking a subsequence) they converge to some functions $\left(S_{c, *}, S_{w, *}, I_{c, *}, E_{w, *}, I_{w, *}, I_{d, *}, V_{*}\right)$ as $n \rightarrow \infty$ in the following topologies:

$$
\begin{aligned}
I_{c, X_{n}} & \rightarrow I_{c, *}, I_{w, X_{n}} \rightarrow I_{w, *} \text { in } C_{l o c}^{1}(\mathbb{R}), \\
S_{c, X_{n}} & \rightarrow S_{c, *}, S_{w, X_{n}} \rightarrow S_{w, *}, E_{w, X_{n}} \rightarrow E_{w, *}, I_{d, X_{n}} \rightarrow I_{d, *}, V_{X_{n}} \rightarrow V_{*} \text { in } C_{l o c}^{2}(\mathbb{R}) .
\end{aligned}
$$

Furthermore, $\left(S_{c, *}, S_{w, *}, I_{c, *}, E_{w, *}, I_{w, *}, I_{d, *}, V_{*}\right)$ satisfy system (2.1) and

$$
\begin{aligned}
& p_{1}(x) \leq S_{c, *}(x) \leq S_{c 0}, p_{2}(x) \leq S_{w, *}(x) \leq S_{w 0}, \\
& \psi_{1}(x) \leq I_{c, *}(x) \leq \min \left\{M_{0}, \phi_{1}(x)\right\}, \\
& \psi_{2}(x) \leq E_{w, *}(x) \leq \min \left\{M_{0}, \phi_{2}(x)\right\}, \psi_{3}(x) \leq I_{w, *}(x) \leq \min \left\{M_{0}, \phi_{3}(x)\right\}, \\
& \psi_{4}(x) \leq I_{d, *}(x) \leq \min \left\{N_{d}, \phi_{4}(x)\right\}, \psi_{5}(x) \leq V_{*}(x) \leq \min \left\{M_{0}, \phi_{5}(x)\right\}
\end{aligned}
$$

for $x \in \mathbb{R}$. In addition, since $S_{c, X_{n}}^{\prime}(x) \leq 0$ and $S_{w, X_{n}}^{\prime}(x)+E_{w, X_{n}}^{\prime}(x) \leq 0$ for $x \in\left[-X_{n}, X_{n}\right]$, we have $S_{c, *}^{\prime}(x) \leq 0$ and $S_{w, *}^{\prime}(x)+E_{w, *}^{\prime}(x) \leq 0$ for $x \in \mathbb{R}$.

Let $S_{c, *}(+\infty)=S_{c}^{\infty}$ and $S_{w, *}(+\infty)+E_{w, *}(+\infty)=S_{w}^{\infty}$. Then there must be $S_{c}^{\infty}<S_{c 0}$ and $S_{w}^{\infty}<S_{w 0}$. In fact, if $S_{c}^{\infty}=S_{c 0}$, it follows that $S_{c, *}(x)=S_{c 0}$ for all $x \in \mathbb{R}$, and hence $I_{c, *}(x) \equiv 0$, a contradiction. Since $\left\|S_{c, *}\right\|_{C^{2}(\mathbb{R})}<+\infty$, the fluctuation lemma implies that $S_{c, *}^{\prime}( \pm \infty)=0$. Hence, we have

$$
\int_{-\infty}^{+\infty}\left[\beta_{c} S_{c, *}(x) V_{*}(x)+\alpha_{c} S_{c, *}(x) I_{c, *}(x) / N_{c, *}(x)\right] d x=\left(s+v_{c}\right)\left(S_{c 0}-S_{c}^{\infty}\right)>0 .
$$


It follows that $\int_{-\infty}^{\infty} I_{c, *}(x) d x<\infty$. By virtue of $\left\|I_{c, *}\right\|_{C^{1}(\mathbb{R})}<+\infty$, we have $I_{c, *}( \pm \infty)=0$, and it turns out that

$$
\int_{-\infty}^{\infty} I_{c, *}(x) d x=\frac{1}{d_{i c}}\left(s+v_{c}\right)\left(S_{c 0}-S_{c}^{\infty}\right) \text { and } I_{c, *}(x) \leq S_{c 0}-S_{c}^{\infty} \forall x \in \mathbb{R} .
$$

Similarly, we can prove that

$$
\begin{gathered}
\int_{-\infty}^{\infty} E_{w, *}(x) d x=\frac{1}{\mu_{w}}\left(s+v_{w}\right)\left(S_{w 0}-S_{w}^{\infty}\right), E_{w, *}( \pm \infty)=0, \\
\int_{-\infty}^{\infty} I_{w, *}(x) d x=\frac{s+v_{w}}{d_{w}}\left(S_{w 0}-S_{w}^{\infty}\right), \\
I_{w, *}( \pm \infty)=0, I_{w, *}(x) \leq \frac{s+v_{w}}{s}\left(S_{w 0}-S_{w}^{\infty}\right) \forall x \in \mathbb{R} .
\end{gathered}
$$

In conclusion, we have established the following

Theorem 3.7. Assume that $\mathcal{R}_{0}=\rho\left(\mathcal{F V}^{-1}\right)>1$. Then for any $s>s^{*}$, where $s^{*}$ is determined by Lemma 3.1, system (1.1) admits a nonnegative traveling wave solution $\left(S_{c, *}(\xi), S_{w, *}(\xi), I_{c, *}(\xi), E_{w, *}(\xi), I_{w, *}(\xi), I_{d, *}(\xi), V_{*}(\xi)\right)$ with $\xi=x+$ st satisfying $S_{c, *}^{\prime}(\xi) \leq 0, S_{c, *}(-\infty)=S_{c 0}, S_{c, *}(+\infty)=S_{c}^{\infty}<S_{c 0}, S_{w, *}^{\prime}(\xi) \leq 0$, $S_{w, *}(-\infty)=S_{w 0}, S_{w, *}(+\infty)=S_{w}^{\infty}<S_{w 0}, I_{c, *}( \pm \infty)=0, I_{d, *}(-\infty)=0$, $\sup _{x \in \mathbb{R}} I_{d, *}(x)<N_{d}, V_{*}(-\infty)=0, \sup _{x \in \mathbb{R}} V_{*}(x)<+\infty$ and (3.31)-(3.34).

Note that if $\left(S_{c}^{\infty}\right)^{2}+\left(S_{w}^{\infty}\right)^{2}>0$, then by the third and fourth equations of (2.1) we have $V_{*}(+\infty)=0$, which yields $V_{*}^{\prime}( \pm \infty)=0$ and $V_{*}^{\prime \prime}( \pm \infty)=0$. Hence, by the last equation of (2.1) we have $I_{d, *}(+\infty)=0$. Unfortunately, we cannot rule out the case where $S_{c}^{\infty}=S_{w}^{\infty}=0$. In such a case, the traveling wave system can be reduced to the following:

$$
\left\{\begin{array}{l}
\left(s+v_{d}\right) I_{d}^{\prime}=\beta_{d}\left(N_{d}-I_{d}\right) V+\alpha_{d}\left(N_{d}-I_{d}\right) I_{d} / N_{d}-\gamma_{d} I_{d}+D_{d} I_{d}^{\prime \prime}, \\
\left(s+v_{v}\right) V^{\prime}=r_{d} I_{d}-\left(d_{v}+d_{n}\right) V+D_{v} V^{\prime \prime} .
\end{array}\right.
$$

Such a system can admit a positive equilibrium

$$
I_{d}=\frac{\frac{\beta_{d} r_{d} N_{d}}{d_{v}+d_{n}}+\alpha_{d}-\gamma_{d}}{\frac{\beta_{d} r_{d} N_{d}}{d_{v}+d_{n}}+\frac{\alpha_{d}}{N_{d}}}, \quad V=\frac{r_{d}}{d_{v}+d_{n}} I_{d}
$$

Therefore, it is possible to have $I_{d, *}(+\infty)>0$ and $V_{*}(+\infty)>0$. It remains an interesting problem for future studies when $I_{d, *}(+\infty)=V_{*}(+\infty)=0$.

We also remark that $s^{*}$ is obtained from the stability analysis of the traveling system in the region $\chi \rightarrow-\infty$ and hence is expected to be the minimal wave speed. As the system involves multiple bird species and a component for environmental contamination (acting like a vector), detailed analysis about the minimal wave speed, its connection with the propagation speed, and the stability of wave solutions is not available at this stage.

\section{REFERENCES}

1. S. Agmon, A. Douglis, L. Nirenberg, Estimates near the boundary for solutions of elliptic differential equations satisfying general boundary conditions. I, Comm. Pure Appl. Math. 12 (1959), 623-727. MR0125307 (23:A2610)

2. S. Agmon, A. Douglis, L. Nirenberg, Estimates near the boundary for solutions of elliptic differential equations satisfying general boundary conditions. II, Comm. Pure Appl. Math. 17 (1964), 35-92. MR0162050(28:5252)

3. A. Berman, M. Neumann, Ronald J. Stern, Nonnegative matrices in dynamic systems, John Wiley \& Sons, New York, 1989. MR.1019319 (90j:93030) 
4. A. Ducrot, P. Magal, Travelling wave solutions for an infection-age structured model with diffusion, Proc. Royal Soc. Edinburgh (A) 139 (2009), 459-482. MR2506782 (2010f:35417)

5. A. Ducrot, P. Magal, S. Ruan, Travelling wave solutions in multigroup age-structured epidemic models, Arch. Rational Mech. Anal. 195 (2010), 311-331. MR2564476

6. D. Gilbarg, N.S. Trudinger, Elliptic partial differential equations of second order, Springer, Berlin, 2001. MR 1814364 (2001k:35004)

7. M. Hirsch, H. Hanish, J.-P. Gabriel, Differential equation model of some parasitic infections: Methods for the study of asymptotic behavior, Comm. Pure Appl. Math. 38 (1985), 733-753. MR:812345 (87f:92015)

8. Y. Hosono, B. Ilyas, Traveling waves for a simple diffusive epidemic model, Math. Models Methods Appl. Sci. 5 (1995), 935-966. MR1359215 (96j:35248)

9. R. Liu, V.R.S.K. Duvvuri, J. Wu, Spread pattern formation of H5N1-avian influenza and its implications for control strategies, Math. Model. Nat. Phenom. 3(7) (2008), 161-179. MR2460260 (2009j:92059)

10. Z.C. Wang, J. Wu, Traveling waves of a diffusive Kermack-McKendrick epidemic model with non-local delayed transmission, Proc. R. Soc. London Ser. A 466 (2010), 237-261. MR2564926 (2011b:92072)

School of Mathematics and Statistics, Lanzhou University, Lanzhou, Gansu, 730000 , People's Republic of China

E-mail address: wangzhch@lzu.edu.cn

Centre for Disease Modeling, York Institute for Health Research and Department of Mathematics and Statistics, York University, Toronto, Ontario, M3J 1P3, Canada

E-mail address: wujh@mathstat.yorku.ca

Department of Mathematics and Department of Zoology and Physiology, University of Wyoming, LaRAmie, Wyoming 82072

E-mail address: Rongsong.Liu@uwyo.edu 